\title{
Pharmacogenetic Association of the Galanin Receptor (GALRI) SNP rs27I7I62 with Smoking Cessation
}

\author{
Allison B Gold', E Paul Wileyto', Adriana Lori' ${ }^{2}$, David Conti ${ }^{3}$, Joseph F Cubells ${ }^{2}$ and Caryn Lerman*,' \\ 'Center for Interdisciplinary Research on Nicotine Addiction, Department of Psychiatry, University of Pennsylvania, Philadelphia, PA, USA; \\ ${ }^{2}$ Department of Human Genetics, Emory University School of Medicine, Atlanta, GA, USA; ${ }^{3}$ Department of Preventive Medicine, \\ University of Southern California, Los Angeles, CA, USA
}

\begin{abstract}
Galanin modulates dopaminergic neurotransmission in the mesolimbic dopamine system, thereby influencing the rewarding effects of nicotine. Variants in the galanin receptor I (GALRI) gene have been associated with retrospective craving severity and heaviness of smoking in prior research. We investigated pharmacogenetic associations of the previously studied GALR/ polymorphism, rs27I7I62, in 1217 smokers of European ancestry who participated in one of three pharmacogenetic smoking cessation clinical trials and were treated with nicotine patch $(n=623)$, nicotine nasal spray $(n=189)$, bupropion $(n=213)$, or placebo $(n=192)$. The primary endpoint was abstinence (7-day point prevalence, biochemically confirmed) at the end of treatment. Cravings to smoke were assessed on the target quit day (TQD). The longitudinal regression model revealed a significant genotype by treatment interaction $(P=0.03)$. There was a reduced odds of quitting success with the presence of at least one minor (C) allele in the bupropion-treated group $(\mathrm{OR}=0.43 ; 95 \%$ $\mathrm{Cl}=0.22-0.77 ; P=0.005)$ but equivalent quit rates by genotype in the nicotine-replacement therapy groups. This genotype by treatment interaction was reproduced in a Cox regression model of time to relapse $(P=0.04)$. In the bupropion trial, smokers carrying the $C$ allele also reported more severe TQD cravings. Further research to identify functional variants in GALRI and to replicate pharmacogenetic associations is warranted.

Neuropsychopharmacology (2012) 37, 1683-1688; doi:10.1038/npp.2012.13; published online 29 February 2012
\end{abstract}

Keywords: nicotine; addiction; craving; pharmacogenetics; galanin receptor

\section{INTRODUCTION}

The neuropeptide galanin is distributed throughout the brain (Melander et al, 1988; Takatsu et al, 2001) and has been implicated in learning and memory (Crawley, 2008; Rustay et al, 2005), feeding behavior (Crawley, 1999), and drug dependence (Picciotto et al, 2010). Galanin modulates mesolimbic dopaminergic neurotransmission (Robinson and Brewer, 2008; Tsuda et al, 1998), and there is evidence that the inhibition of midbrain DA activity by galanin may be mediated, in part, via the galanin receptor 1 (GALR1) receptor subtype (Counts et al, 2002). Galanin, acting through its receptor subtypes, has been shown in rodent models to modulate reward and withdrawal responses from drugs of abuse (Hawes et al, 2008; Zachariou et al, 1999). Of relevance to nicotine dependence, mice lacking the galanin peptide display decreased sensitivity to nicotine reward (Neugebauer et al, 2011). Further, galanin, mediated via

\footnotetext{
*Correspondence: Dr C Lerman, Center for Interdisciplinary Research on Nicotine Addiction, Department of Psychiatry, University of Pennsylvania, 3535 Market Street, Suite 4100, Philadelphia, PA 19104, USA, Tel: + I 2157467141 , Fax: + I 215746 7140, E-mail: clerman@upenn.edu

Received 21 October 2011; revised 15 January 2012; accepted 18 January 2012
}

GALR1, blocks nicotine reward and reverses physical nicotine withdrawal signs (Jackson et al, 2011).

Translating these preclinical observations, two recent studies have explored the role of variation in the GALR1 gene in nicotine-dependent phenotypes. In European- and African-American smokers, multiple single-nucleotide polymorphism (SNP) markers in the GALR1 gene were associated with self-reported heavy smoking (Jackson et al, 2011). In addition, an analysis of treatment-seeking smokers identified a highly significant association of a single GALR1 SNP, rs2717162, with retrospective reports of tobacco craving during a previous quit attempt (Lori et al, 2011).

Extending these observations, we examined the association of rs2717162 in GALR1 to prospectively assessed smoking cessation and tobacco craving in 1217 smokers of European ancestry who participated in one of three pharmacogenetic smoking cessation clinical trials: (1) a bupropion placebo-controlled randomized trial $(n=405)$ (Lerman et al, 2006); (2) an open-label trial of nicotine patch $(n=441)$ (Schnoll et al, 2010); and (3) a randomized open-label trial of nicotine patch $v s$ nicotine nasal spray $(n=371)$ (Lerman et al, 2006; Lerman et al, 2004). Data were pooled across trials to test for the presence of a genotype by treatment interaction on quitting success, 
time to relapse, and subjective craving on the first day of abstinence.

\section{SUBJECTS AND METHODS}

\section{Participants and Procedures by Clinical Trial}

\section{Bupropion trial}

Sample. Treatment-seeking smokers responding to media advertisements were screened for eligibility from April 1999 to October 2001 at Georgetown University (Washington, DC) and SUNY Buffalo (New York). Inclusion criteria were: ages 18-65 and a smoking rate of $\geqslant 10$ cigarettes a day for the prior 12 months. Exclusion criteria included a history of DSM-IV axis I psychiatric disorder, seizure disorder, current use of psychotropic medications, and pregnancy or lactation. In the full cohort of smokers, there were 555 trial participants, including 436 of self-reported European ancestry. Of these smokers, DNA was available for 428 , and GALR1 genotyping was completed for 405 subjects (failed SNP assays and DNA samples with low call rates were removed from the data set after confirming replicate concordance). Prior analyses of ancestry informative markers in this sample revealed no evidence for population stratification (Lerman et al, 2006). Of the 405 eligible participants, $55 \%$ were female, $46 \%$ were college graduates, and the average age was $44.4(\mathrm{SD}=11.6)$. On average, participants smoked 22 cigarettes per day $(S D=9.4)$. The mean score on the Fagerström Test for Nicotine Dependence (FTND) was $5.1(\mathrm{SD}=2.1)$.

Procedures. The study was approved by the University of Pennsylvania and SUNY Buffalo institutional review boards. Participants at both sites received identical assessments of demographics, smoking rate, and nicotine dependence assessed with the FTND (Heatherton et al, 1991) and provided a blood sample for genotyping. All participants received 10 weeks of either bupropion or matched placebo, initiated 2 weeks prior to the target quit date following a pre-quit counseling session. Bupropion was administered according to the guidelines $(150 \mathrm{mg} /$ day $\times$ 3 days, followed by $300 \mathrm{mg} /$ day). Participants received seven sessions of standardized behavioral group counseling during the medication phase. Self-reported smoking was assessed using the time-line follow-back procedure (Brown et al, 1998). Consistent with prior pharmacogenetic trials (Lerman et al, 2006; Ray et al, 2010), the primary outcome was biochemically verified (saliva cotinine $\leqslant 15 \mathrm{ng} / \mathrm{ml}$ ) 7-day point-prevalence abstinence at the end of treatment (EOT) and 6-months post target quit date. As per convention (SRNT, 2002), participants who reported smoking within 7 days before the assessment, failed to provide a cotinine sample, or provided a cotinine sample $>15 \mathrm{ng} / \mathrm{ml}$ were considered non-abstinent.

\section{Nicotine patch trial}

Sample. Treatment-seeking smokers were screened at the University of Pennsylvania (Philadelphia, PA) from 2004 to 2008, using methods and inclusion criteria similar to those described above. In the full cohort of 568 trial participants, there were 478 smokers of self-reported European ancestry. Of these, DNA was available for 451 and GALR1 genotyping was completed for 441 subjects (failed SNP assays and DNA samples with low call rates were removed from the data set after confirming replicate concordance). Prior analyses of ancestry informative markers in this sample revealed no evidence for population stratification (Ray et al, 2010). Of the final 441 participants, $42 \%$ were female, $35 \%$ were college graduates, and the average age was $44.9(\mathrm{SD}=10.4)$. On average, participants smoked 22 cigarettes per day $(\mathrm{SD}=9.0)$. The mean score on the FTND was $5.3(\mathrm{SD}=2.2)$. Thus, this sample was highly comparable to the bupropion trial sample.

Procedures. The study protocol was approved by the University of Pennsylvania institutional review board. Participants completed pretreatment assessments identical to those described above for the bupropion trial. After a pre-quit counseling session, transdermal nicotine-patch therapy was initiated on the target quit date and continued for 8 weeks; all participants received three sessions of behavioral counseling during the treatment period (Schnoll et al, 2010). The primary outcome was 7-day point-prevalence abstinence at the end of 8-week treatment. The only difference in outcome assessment is that self-reported smoking for the 7 days prior to the EOT survey was biochemically verified using exhaled breath carbon monoxide (CO) (cotinine verification was not used because the treatment contained nicotine). The primary outcome was biochemically confirmed 7-day point-prevalence abstinence at the EOT. As per convention (SRNT, 2002), participants who reported smoking within 7 days before the assessment, failed to provide a $\mathrm{CO}$, or provided a $\mathrm{CO}>10$ p.p.m were considered nonabstinent.

\section{Nicotine patch vs nicotine spray trial}

Sample. Treatment-seeking smokers were recruited at Georgetown University and the University of Pennsylvania (Philadelphia, PA), from February 2000 to August 2003, using methods identical to those described above. In the full cohort of 600 trial participants, there were 397 smokers of self-reported European ancestry. Of these, DNA was available and GALR1 genotyping was completed for 371 participants (nicotine patch, $n=182$; nicotine nasal spray, $n=189$ ) (failed SNP assays and DNA samples with low call rates were removed from the data set after confirming replicate concordance). Prior analyses of ancestry informative markers in this sample revealed no evidence for population stratification (Lerman et al, 2006 ). Of the 371 participants, $47 \%$ were female, $52 \%$ were college graduates, and the average age was 46.3 $(\mathrm{SD}=11.5)$. On average, participants smoked 23 cigarettes per day ( $\mathrm{SD}=9.3)$. The mean score on the FTND was $5.5(\mathrm{SD}=2.2)$.

Procedures. The institutional review boards from both universities approved the research protocols. The procedures and outcome assessments were identical to those described above for the nicotine-patch trial, except that participants were randomized to nicotine patch or nicotine nasal spray, and 7 sessions of behavioral group counseling were delivered during the 8-week treatment period. As per convention (SRNT, 2002), participants who reported smoking within 7 days before the assessment, failed to provide a $\mathrm{CO}$, or provided a $\mathrm{CO}>10$ p.p.m were considered non-abstinent. 


\section{Genotyping}

Genotyping for the GALR1 SNP rs2717162 was completed using the ABI Prism 7900HT Sequence Detection System (Applied Biosystems, Foster City, CA). PCR was performed with $2.25 \mathrm{ng}$ of DNA, $2.5 \mu \mathrm{l}$ of ABI Taqman Universal Mastermix, $0.125 \mu \mathrm{l}$ of water, and $0.125 \mu \mathrm{l}$ of $40 \mathrm{X}$ Assay by Design SNP Assay for the GALR1 variant (ABI, Foster City, CA). The 5- $\mu$ l reactions were performed in a 384-well plate (ABI). The plates were thermal cycled using the same conditions described previously (Lerman et al, 2006). The plates were scanned utilizing the Allelic Discrimination End-Point Analysis on the ABI Prism 7900HT Sequence Detection System. The allelic discrimination data were analyzed by the AutoCall algorithm of the SDS v2.1 Software (ABI).

\section{Craving assessment}

In all the three trials, craving was assessed as the sum of two items from a withdrawal symptom checklist, validated in previous research (Killen and Fortmann, 1997; Wang et al, 2007). On a Likert scale $(0=$ not present, $1=$ mild, $2=$ moderate, and $3=$ severe), participants rated cravings for cigarettes and urges to smoke. For this analysis, we focused on cravings reported on the target quit date in biochemically verified abstainers at the target quit day (TQD) visit.

\section{Statistical analysis}

Our outcomes of interest were point-prevalence abstinence (binary) at 8 weeks and 6 months, time to first smoking lapse (continuous), and craving (continuous) at TQD. Preliminary analyses of bivariate associations of outcomes with genotype were conducted using contingency tables $\left(\chi^{2}\right), t$-tests, or oneway ANOVA as appropriate. To estimate pharmacogenetic associations, data were pooled across trials; there were no significant differences in genotype, demographic, or smoking history variables by treatment arm. The primary endpoint, point-prevalence abstinence, was analyzed by longitudinal logistic regression, estimated using generalized estimating equation methods. The model included the GALR1 rs2717162 genotype (dichotomized, $\mathrm{TT}=0, \mathrm{C}^{*}=1$ ), treatment group, time point, and time $\times$ treatment interactions. We also included sex and nicotine dependence level as controlling variables and tested treatment $\times$ genotype interactions for inclusion. We analyzed time to first cigarette (censored at week 8) using Cox regression. Finally, we analyzed the effect of genotype on quit-day craving (urge to smoke) using multiple linear regression, adjusted for sex, nicotine dependence score, genotype, and treatment. All analyses were conducted using STATA (Stata Corporation, College Station, TX). With respect to statistical power, the 410 subjects in the bupropion trial yielded $80 \%$ power to detect a $13 \%$ difference in quit rates by genotype. The detectable difference was $18 \%$ for nicotine spray and $10 \%$ for the combined patch trials.

\section{RESULTS}

\section{Descriptive Data}

Descriptive data below are presented by treatment arm consistent with the pooled analysis estimating genotype effects across treatments.
Bupropion. For the rs2717162 SNP, 135 (63.4\%) participants were homozygous for the T allele (TT), 68 (31.9\%) were heterozygous (CT), and $10(4.7 \%)$ were homozygous for the $\mathrm{C}$ allele (CC). The rs2717162 genotypes were in Hardy-Weinberg equilibrium $(P=0.71)$. There were no significant differences in demographics, FTND, cigarettes per day, or treatment group assignment between genotype groups $(P>0.10)$.

Placebo. For the rs2717162 SNP, 104 (54.2\%) participants were homozygous for the $\mathrm{T}$ allele (TT), 76 (39.6\%) were heterozygous (CT), and $12(6.2 \%)$ were homozygous for the $\mathrm{C}$ allele (CC). The rs2717162 genotypes were in Hardy-Weinberg equilibrium $(P=0.70)$. There were no significant differences in demographics, FTND, cigarettes per day, or treatment group assignment between genotype groups $(P>0.10)$.

Nicotine-patch treatment. For the rs2717162 SNP, 366 (58.7\%) participants were homozygous for the T allele (TT), $226(36.3 \%)$ were heterozygous (CT), and 31 (5.0\%) were homozygous for the $\mathrm{C}$ allele (CC). The rs2717162 genotypes were in Hardy-Weinberg equilibrium $(P=0.61)$. There were no significant differences in demographics, FTND, or cigarettes per day between genotype groups $(P>0.10)$.

Nicotine-spray treatment. For the rs2717162 SNP, 110 (58.2\%) participants were homozygous for the T allele (TT), $66(34.9 \%)$ were heterozygous (CT), and 13 (6.9\%) were homozygous for the $\mathrm{C}$ allele (CC). The rs2717162 genotypes were in Hardy-Weinberg equilibrium $(P=0.48)$. There were no significant differences in demographics, FTND, or cigarettes per day between genotype groups $(P>0.10)$.

\section{Abstinence}

At the end of 8 weeks, $367(30.1 \%)$ participants were abstinent. At the 6-month follow-up, 268 (22.0\%) participants were abstinent. Abstinence rates by genotype and treatment are shown in Figure 1. The results of our longitudinal regression model of abstinence at EOT and 6month follow-up revealed a significant genotype by treatment interaction $(P=0.03)$; there was a reduced odds of quitting success with the presence of at least one minor $(C)$ allele in the bupropion-treated group only $(\mathrm{OR}=0.43 ; 95 \%$ $\mathrm{CI}=0.22-0.77 ; P=0.005)$. There was no interaction of genotype by time point (EOT vs $6 \mathrm{M}$ ). Likewise, time to selfreported continuous abstinence failure using Cox regression revealed a significant genotype by treatment interaction $(P=0.04)$ and an association of the $C$ allele with faster time to smoking relapse in the bupropion-treated group $(\mathrm{HZ}=2.33 ; 95 \% \mathrm{CI}=1.30-4.55 ; P=0.005)$ (Figure 2$)$.

\section{Craving}

In the bupropion trial, where significant associations of the $\mathrm{C}$ allele with relapse were observed, craving scores of abstainers on TQD $(n=264$, saliva continine $<15 \mathrm{ng} / \mathrm{ml})$ were higher in the $\mathrm{C}$-allele group $(\mathrm{M}=5.78 ; \mathrm{SD}=1.40)$ compared with the TT group $(\mathrm{M}=5.32 ; \mathrm{SD}=1.43 ; \mathrm{F}(1,262)=6.85 ; P=0.009)$. Results of the linear regression model of TQD craving, controlling for effect of treatment, revealed a significant genotype association for each copy of the risk allele $(t(267)=3.35 ; P=0.001)$. Craving predicted reduced likelihood of abstinence $(\mathrm{OR}=0.78 ; 95 \% \quad \mathrm{CI}=0.67-0.92$; $P=0.002$ ). However, inclusion of craving in the model did not have a meaningful effect on the genotype association with 


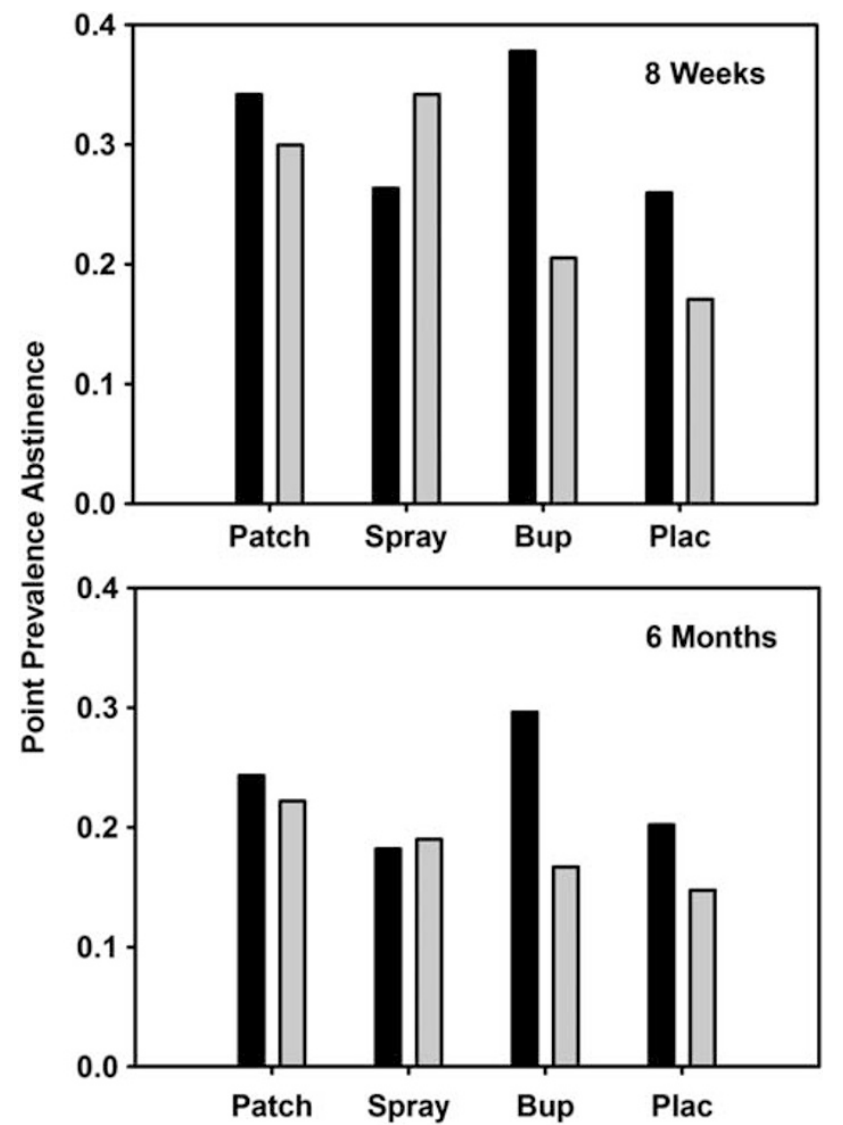

Figure I Biochemically verified 7-day point-prevalence abstinence rates at 8 weeks and 6 months by genotype and treatment arm. There was a significant genotype by treatment interaction $(P=0.03)$. Among participants in the bupropion trial, those carrying a minor (C) allele (gray bars) had significantly lower quit rates at the EOT compared with TT homozygotes (black bars), independent of treatment arm assignment $(\mathrm{OR}=0.43 ; 95 \% \mathrm{Cl}=0.22-0.77 ; P=0.005)$. Among $C$ allele carriers, quit rates differed in post-hoc testing $\left(\chi^{2}=9.32 ; P=0.025\right)$.

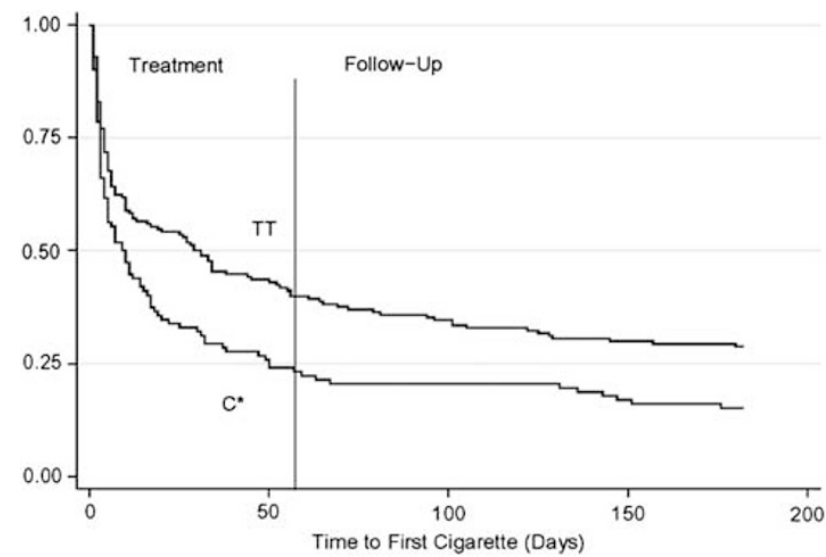

Figure 2 (Bupropion treatment) Relapse hazard for time to first cigarette. Subjects carrying at least one $C$ allele relapsed significantly more quickly from TQD to EOT $(P=0.005)$, reported as time to first cigarette.

abstinence (OR changed from 0.52 to 0.56 ), suggesting that genotype associations are not mediated by craving. There were no significant genotype associations with craving in the nicotine-replacement therapy trials (all $P$-values $>0.10$ ).

\section{DISCUSSION}

This study examined the rs2717162 SNP in the GALR1 gene for associations with smoking cessation and treatment response in a pooled analysis of 1217 smokers of European ancestry participating in one of three pharmacogenetic clinical trial cohorts. The presence of a genotype by treatment interaction indicated significant heterogeneity in genotype associations with abstinence in the different treatment arms. There was a reduced odds of quitting success and a faster rate of relapse with the presence of at least one minor (C) allele in the bupropion-treated group but not in the placebo, nicotine patch, or nicotine nasal spray groups.

Consistent with the observed pharmacogenetic association, among smokers treated with bupropion, the presence of at least one minor (C) allele was also associated with more severe cravings on the target quit date. In a recent study (Lori et al, 2011), the rs2717162 SNP was associated with self-reported cravings to smoke as experienced during a prior quit attempt. Although study participants were treatment-seeking smokers, the craving assessment was performed at baseline and was retrospective. Individuals carrying the $\mathrm{T}$ allele had higher craving scores than those carrying the minor $\mathrm{C}$ allele, which is the opposite of what we observed for target quit-date cravings in the bupropion trial. A second study (Jackson et al, 2011) used a recombinant inbred strain mouse model and human genetic approach to investigate the role of GALR1 in nicotine dependence. In the mouse model, somatic signs of withdrawal were correlated with GALR1 expression in the nucleus accumbens. In the human smoker cohorts, two GALR1 variants were associated with heavy smoking, based on the reported number of cigarettes smoked per day. Data available from HAPMAP (hapmap.org) show that one of the significant markers, rs2717164, is in linkage disequilibrium (LD) with rs2717162. However, we did not find an association of this SNP with heavy smoking in our cohorts (results not shown).

The divergence of the findings for cravings in the current trial and prior studies are likely due to differences in study design and phenotype assessment. The current study assessed craving and smoking cessation prospectively because retrospective reports of prior quit attempts can be subject to bias (Shiffman et al, 2008). Further, the current pharmacogenetic analysis indicates that genotype associations are modified by smoking-cessation treatment. In addition, the SNPs examined by the prior study and our study are not known to be functional, thus, associations may be due to a rare functional variant in LD with rs2717162 (Bodmer and Bonilla, 2008). A more complete annotation of SNPs in the GALR1 gene could be generated through re-sequencing and functional studies to identify potential causative variants associated with smokingrelated phenotypes.

Although the mechanisms underlying the observed associations of GALR1 variation with craving and abstinence are largely unknown, there is a biological rationale to support a potential contribution of GALR1 to these phenotypes. Galanin and GALR1 are found in cortico-limbic regions (Holmes et al, 2003) and galanin receptors are highly expressed on neuronal terminals in striatum and nucleus accumbens (Hawes et al, 2008). These regions and 
circuits are important in abstinence-induced cravings (Wang et al, 2007). In addition, galanin is co-expressed with acetylcholine in the paraventricular nucleus (PVN), and galanin injection into the PVN can cause release of dopamine and inhibit acetylcholine release in the nucleus accumbens (Rada et al, 1998). Acetylcholine acts on nAChRs located on glutamate and GABA neuronal terminals in the VTA, which project to the nucleus accumbens, amygdala, and prefrontal cortex (Kalivas and Volkow, 2005). Thus, galanin may exert its effects on dopamine release through the cholinergic system (Ericson and Ahlenius, 1999; Picciotto, 2010).

The observed pharmacogenetic effect is also biologically plausible, but alternate interpretations are speculative at this time. Although the results indicate that the genotype association is significant in the bupropion arm only, visual inspection of Figure 1 reveals a similar trend for the placebo arm; in both arms, C-allele carriers have higher relapse rates. Thus, it is less likely that the results reflect an enhanced response to bupropion in smokers homozygous for the $\mathrm{T}$ allele. Another possibility is that there is a liability to relapse in the $\mathrm{C}$ allele carriers that is not modified by bupropion (or placebo) but is reversed by nicotine-replacement therapy. Indeed, inspection of Figure 1 shows higher quit rates in the $\mathrm{C}$ allele carriers treated with nicotine patch or nicotine spray relative to placebo or bupropion. Although nicotine treatment (nicotine patch or spray) has agonist effects at the nAChRs, bupropion therapy has antagonist effects (Slemmer et al, 2000). An agonist at nAChRs may complement the effects of GALR1 on dopamine-mediated neurotransmission but an antagonist blocks the effects of nicotine and may not offset the involvement of GALR1.

There are limitations of this study that should be considered. Although the study sample of 1217 participants is larger than most prior pharmacogenomic analyses and was amply powered to detect genotype by treatment effects, the number of individuals homozygous for the minor allele was relatively small; thus, we dichotomized the genotype variable. Importantly, the functional consequences of rs2717162 are unknown and it is likely that the associations observed in this study and prior studies are due to other SNPs in the GALR1 gene. We selected only one SNP based on the strong genetic association with retrospective craving observed previously (Lori et al, 2011).

Preclinical studies that alter the expression and activity of GALR1 and analyze a broader array of dependenceassociated behavioral phenotypes in rodents would be useful to increase our understanding of the role of galanin and the GALR1 in smoking cessation. Re-sequencing of GALR1 would be valuable to identify the functional variants that may be contributing to craving and abstinence. If further investigations confirm the association of GALR1 with nicotine dependence and smoking cessation, this could lead to the development of novel therapeutics to aid in smoking cessation.

\section{ACKNOWLEDGEMENTS}

This study was supported by the following Grants: NIH P50CA-143187 (CL) and NIH U01-DA-020830 (CL).

\section{DISCLOSURE}

Dr Caryn Lerman has served as a consultant and/or has received research funding from GlaxoSmithKline, AstraZeneca, Novartis, and Pfizer. The current study was not supported by industry funds. Drs E Paul Wileyto and David Conti have served as consultants for Pfizer. Dr Joseph F Cubells has received grant support from NIMH, NARSAD, NIDA, The Robert W Woodruff Fund of the Emory University, Roche, and Seaside Therapeutics, and has consulted for Dykema, Gossett, LLP, and Barnes and Thornburg, LLP. The remaining authors have no conflict of interest.

\section{REFERENCES}

Bodmer W, Bonilla C (2008). Common and rare variants in multifactorial susceptibility to common diseases. Nat Genet 40: 695-701.

Brown RA, Burgess ES, Sales SD, Whiteley JA, Evans DM, Miller IW (1998). Reliability and validity of a smoking timeline followback interview. Psychol of Addict Behav 12: 101-112.

Counts SE, McGuire SO, Sortwell CE, Crawley JN, Collier TJ, Mufson EJ (2002). Galanin inhibits tyrosine hydroxylase expression in midbrain dopaminergic neurons. J Neurochem 83: $442-451$

Crawley JN (1999). The role of galanin in feeding behavior. Neuropeptides 33: 369-375.

Crawley JN (2008). Galanin impairs cognitive abilities in rodents: relevance to Alzheimer's disease. Cell Mol Life Sci 65: 1836-1841.

Ericson E, Ahlenius S (1999). Suggestive evidence for inhibitory effects of galanin on mesolimbic dopaminergic neurotransmission. Brain Res 822: 200-209.

Hawes JJ, Brunzell DH, Narasimhaiah R, Langel U, Wynick D, Picciotto MR (2008). Galanin protects against behavioral and neurochemical correlates of opiate reward. Neuropsychopharmacology 33: 1864-1873.

Heatherton TF, Kozlowski LT, Frecker RC, Fagerstrom KO (1991). The Fagerstrom test for nicotine dependence: a revision of the fagerstrom tolerance questionnaire. $\mathrm{Br} J$ Addict 86: $1119-1127$.

Holmes A, Heilig M, Rupniak NM, Steckler T, Griebel G (2003). Neuropeptide systems as novel therapeutic targets for depression and anxiety disorders. Trends Pharmacol Sci 24: 580-588.

Jackson KJ, Chen X, Miles MF, Harenza J, Damaj MI (2011). The Neuropeptide Galanin and Variants in the GalR1 Gene are Associated with Nicotine Dependence. Neuropsychopharmacology 36: 2339-2348.

Kalivas PW, Volkow ND (2005). The neural basis of addiction: a pathology of motivation and choice. Am J Psychiatry 162: 1403-1413.

Killen JD, Fortmann SP (1997). Craving is associated with smoking relapse: findings from three prospective studies. Exp Clin Psychopharmacol 5: 137-142.

Lerman C, Jepson C, Wileyto EP, Epstein LH, Rukstalis M, Patterson F et al (2006). Role of functional genetic variation in the dopamine D2 receptor (DRD2) in response to bupropion and nicotine replacement therapy for tobacco dependence: results of two randomized clinical trials. Neuropsychopharmacology 31: 231-242.

Lerman C, Kaufmann V, Rukstalis M, Patterson F, Perkins K, Audrain-McGovern $\mathrm{J}$ et al (2004). Individualizing nicotine replacement therapy for the treatment of tobacco dependence: a randomized trial. Ann Intern Med 140: 426-433.

Lori A, Tang Y, O’Malley S, Picciotto MR, Wu R, Conneely KN et al (2011). The galanin receptor 1 gene associates with tobacco craving in smokers seeking cessation treatment. Neuropsychopharmacology 36: 1412-1420. 
Pharmacogenetic association of rs27 I7 I62 with smoking $A B$ Gold et al

Melander T, Kohler C, Nilsson S, Hokfelt T, Brodin E, Theodorsson $\mathrm{E}$ et al (1988). Autoradiographic quantitation and anatomical mapping of 125I-galanin binding sites in the rat central nervous system. J Chem Neuroanat 1: 213-233.

Neugebauer NM, Henehan RM, Hales CA, Picciotto MR (2011). Mice lacking the galanin gene show decreased sensitivity to nicotine conditioned place preference. Pharmacol Biochem Behav 98: 87-93.

Picciotto MR (2010). Galanin and addiction. EXS 102: 195-208.

Picciotto MR, Brabant C, Einstein EB, Kamens HM, Neugebauer NM (2010). Effects of galanin on monoaminergic systems and HPA axis: Potential mechanisms underlying the effects of galanin on addiction- and stress-related behaviors. Brain Res 1314: 206-218.

Rada P, Mark GP, Hoebel BG (1998). Galanin in the hypothalamus raises dopamine and lowers acetylcholine release in the nucleus accumbens: a possible mechanism for hypothalamic initiation of feeding behavior. Brain Res 798: 1-6.

Ray R, Mitra N, Baldwin D, Guo M, Patterson F, Heitjan DF et al (2010). Convergent evidence that choline acetyltransferase gene variation is associated with prospective smoking cessation and nicotine dependence. Neuropsychopharmacology 35: 1374-1382.

Robinson JK, Brewer A (2008). Galanin: a potential role in mesolimbic dopamine-mediated instrumental behavior. Neurosci Biobehav Rev 32: 1485-1493.

Rustay NR, Wrenn CC, Kinney JW, Holmes A, Bailey KR, Sullivan TL et al (2005). Galanin impairs performance on learning and memory tasks: findings from galanin transgenic and GAL-R1 knockout mice. Neuropeptides 39: 239-243.

Schnoll RA, Patterson F, Wileyto EP, Heitjan DF, Shields AE, Asch DA et al (2010). Effectiveness of extended-duration transdermal nicotine therapy: a randomized trial. Ann Intern Med 152: 144-151.

Shiffman S, Brockwell SE, Pillitteri JL, Gitchell JG (2008). Use of smoking-cessation treatments in the United States. Am J Prev Med 34: 102-111.

Slemmer JE, Martin BR, Damaj MI (2000). Bupropion is a nicotinic antagonist. J Pharmacol Exp Ther 295: 321-327.

SRNT (2002). Biochemical verification of tobacco use and cessation. Nicotine Tob Res 4: 149-159.

Takatsu Y, Matsumoto H, Ohtaki T, Kumano S, Kitada C, Onda H et al (2001). Distribution of galanin-like peptide in the rat brain. Endocrinology 142: 1626-1634.

Tsuda K, Tsuda S, Nishio I, Masuyama Y, Goldstein M (1998). Effects of galanin on dopamine release in the central nervous system of normotensive and spontaneously hypertensive rats. Am J Hypertens 11: 1475-1479.

Wang Z, Faith M, Patterson F, Tang K, Kerrin K, Wileyto EP et al (2007). Neural substrates of abstinence-induced cigarette cravings in chronic smokers. J Neurosci 27: 14035-14040.

Zachariou V, Parikh K, Picciotto MR (1999). Centrally administered galanin blocks morphine place preference in the mouse. Brain Res 831: 33-42. 